\title{
Acolhimento e classificação de risco: percepção de profissionais de saúde e usuários
} User embracement and risk classification: health professionals' and users' perceptions Acogida y clasificación de riesgo: percepción de profesionales sanitarios y usuarios

\author{
Thais Santos Campos iD \\ Hospital São Vicente de Paulo - Cruz Alta (RS) - Brasil \\ Éder Luís Arboit iD \\ Universidade de Cruz Alta - UNICRUZ - Cruz Alta (RS) - Brasil \\ Claudelí Mistura iD \\ Universidade de Cruz Alta - UNICRUZ - Cruz Alta (RS) - Brasil \\ Cristina Thum iD \\ Universidade de Cruz Alta - UNICRUZ - Cruz Alta (RS) - Brasil \\ Jaqueline Arboit iD \\ Universidade Federal de Santa Maria - UFSM - Santa Maria (RS) - Brasil

\section{Silviamar Camponogara (iD} \\ Universidade Federal de Santa Maria - UFSM - Santa Maria (RS) - Brasil
}

\section{RESUMO}

Objetivo: Conhecer a percepção de profissionais de saúde e usuários em relação ao acolhimento com classificação de risco em um serviço de urgência/emergência. Métodos: Estudo exploratóriodescritivo, com abordagem qualitativa, realizado em uma unidade de urgência/emergência de um hospital do interior do Rio Grande do Sul, Brasil. A coleta de dados ocorreu em agosto e setembro de 2017, através de entrevista semiestruturada realizada com 15 profissionais de saúde e nove usuários do serviço. A análise obedeceu aos pressupostos da análise de conteúdo, emergindo três categorias temáticas: $O$ cotidiano de trabalho dos profissionais em relação ao acolhimento e classificação de risco; Dificuldade do usuário em compreender a classificação de risco estabelecida pelos profissionais; Importância da capacitação para atuação em equipe no acolhimento com classificação de risco. Resultados: Os usuários possuem pouco conhecimento sobre o acolhimento com classificação de risco, assim como sobre o funcionamento do mesmo. Os profissionais não se sentem preparados para trabalhar com esse sistema em função da falta de treinamento adequado, por vezes classificando os usuários de forma inadequada, o que pode agravar o quadro clínico e o prognóstico, além de dificultar a efetivação da integralidade do cuidado. Conclusão: Evidenciou-se que os usuários não têm clareza a respeito dos critérios utilizados para a realização do Protocolo de acolhimento com classificação de risco, gerando conflitos entre profissionais e usuários. Há necessidade de se repensar as formas de esclarecer os usuários quanto à importância desse sistema.

Descritores: Acolhimento; Classificação; Risco; Socorro de Urgência; Emergências; Enfermagem.

\section{ABSTRACT}

Objective: To know health professionals' and users' perceptions of user embracement and risk classification in an urgency/ emergency service. Methods: A qualitative exploratory descriptive study was conducted in an urgency/emergency center of a hospital in the countryside of Rio Grande do Sul, Brazil. Data were collected in August and September 2017 through semi-structured interviews with 15 health professionals and nine service users. The analysis followed the assumptions of content analysis and three thematic categories emerged: Professionals' daily work in relation to user embracement and risk classification; User's difficulty in understanding the risk classification made by professionals; Importance of training for teamwork in user embracement with risk classification. Results: The users have little knowledge about user embracement with risk classification and its functioning. The

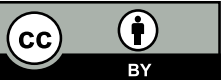


professionals do not feel prepared to work with this system due to lack of adequate training and sometimes end up inappropriately classifying the users, which may worsen the clinical picture and prognosis in addition to hindering the delivery of comprehensive care. Conclusion: The users do not understand clearly the criteria adopted to carry out the user embracement with risk classification protocol, which generates conflicts between professionals and users. There is a need to rethink ways to inform users about the importance of this system.

Descriptors: User Embracement; Classification; Risk; Emergency Relief; Emergencies; Nursing.

\section{RESUMEN}

Objetivo: Conocer la percepción de profesionales sanitarios y usuarios respecto la acogida con la clasificación de riesgo de un servicio de urgencia/emergencia. Métodos: Estudio exploratoriodescriptivo de abordaje cualitativo realizado en una unidad de urgencia/emergencia de un hospital de Rio Grande do Sul, Brasil. La recogida de datos se dio entre agosto y septiembre de 2017 a través de entrevista semiestructurada realizada con 15 profesionales sanitarios y nueve usuarios del servicio. El análisis obedeció a los presupuestos del análisis de contenido del cual emergieron tres temáticas: El cotidiano de trabajo de los profesionales respecto la acogida y la clasificación de riesgo; Dificultad del usuario de comprender la clasificación de riesgo establecida por los profesionales; Importancia de un equipo preparado para la acogida con clasificación de riesgo. Resultados: Los usuarios tienen poco conocimiento sobre la acogida con clasificación de riesgo así como el funcionamiento del mismo. Los profesionales no están preparados para trabajar con ese sistema por no tener entrenamiento adecuado, algunas veces clasificando los usuarios de manera inadecuada lo que puede empeorar el cuadro clínico y el pronóstico además de dificultar la efectuación de la integralidad del cuidado. Conclusión: Se ha evidenciado que los usuarios no están seguros de los criterios utilizados para la realización del protocolo de acogida con clasificación de riesgo lo que genera conflictos entre profesionales y usuarios. Hay la necesidad de repensar las formas de aclarar los usuarios sobre la importancia de ese sistema.

Descriptores: Acogimiento; Clasificación; Riesgo; Socorro de Urgencia; Urgencias Médicas; Enfermería.

\section{INTRODUÇÃO}

O atendimento de urgência/emergência constitui uma forma diferenciada de assistência à saúde, cujas decisões, na maioria das vezes, são tomadas em curto espaço de tempo. Nesse sentido, a Rede de Atenção às Urgências (RAU) precisa articular suas ações nos três níveis de atenção à saúde, no intuito de ampliar e qualificar o acesso humanizado e integral aos usuários que necessitam desses atendimentos ${ }^{(1)}$.

Os serviços de pronto atendimento são reconhecidos como "portas de entrada" do Sistema Único de Saúde (SUS) e, por vezes, possuem alta demanda e superlotação, o que dificulta a identificação das prioridades de atendimento. Entre os fatores relacionados a essa superlotação, destacam-se: o fato de ser utilizado como primeira escolha para obter atendimento em saúde; o aumento da população demográfica e da expectativa de vida, e a prevalência de doenças crônicas ou a procura de atendimento na fase agudas dessas ${ }^{(2)}$.

Diante dessa realidade, o Ministério da Saúde (MS) implantou, em 2009, o Programa de Acolhimento Com Classificação de Risco (ACCR) que consiste num processo dinâmico de identificação e priorização do atendimento, o qual visa a discernir os casos críticos dos não críticos $^{(3)}$. Entre os instrumentos utilizados, mundialmente, para a avaliação do usuário, destacam-se: o inglês Manchester Triage System (Protocolo de Manchester - MTS), o australiano Australasian Triage Scale (ATS), o canadense Canadian Triage and Acuity Scale (CTAS) e o americano Emergency Severity Index (ESI) ${ }^{(4)}$.

No Brasil, a assistência de urgência/emergência é realizada nas Unidades de Pronto Atendimento (UPA), Estratégia Saúde da Família (ESF), Atendimento Pré-Hospitalar (APH) e na área hospitalar na unidade de pronto socorro ou unidade de urgência/emergência. Nesses espaços, o protocolo de Manchester é um dos instrumentos utilizados para a avalição do usuário. Ele também está em uso em outros países, como Holanda, Suécia, Espanha, Portugal e Inglaterra. É constituído por fluxogramas que auxiliam os profissionais na identificação das queixas relatadas pelos usuários e na definição de condutas a serem adotadas ${ }^{(5)}$.

O usuário é classificado em um dos cinco níveis de acordo com as prioridades: emergência, que tem como distintivo a cor vermelha e tempo de espera de zero minuto; muito urgente, usa-se a cor laranja e tempo de espera de até dez minutos; urgente, o distintivo tem cor amarela e o tempo máximo de espera de até sessenta minutos; pouco urgente, de cor verde e com tempo de espera de até cento e vinte minutos, e não urgente, a cor azul com o tempo de espera de até duzentos e quarenta minutos. Para cada nível é estabelecido um tempo para o atendimento médico e para a reavaliação pelo enfermeiro(6). 
A avaliação do quadro clínico deve ocorrer por profissional capacitado, não excedendo cinco minutos. Essa avaliação envolve uma combinação de dados oriundos da identificação dos problemas apresentados pelo usuário e de evidências constatadas a partir da análise do seu estado geral. Trata-se, portanto, de uma instrumento baseado em sinais e sintomas de alerta, a fim de permitir a classificação por níveis de gravidade ${ }^{(6)}$.

O acolhimento com classificação de risco (ACCR) deve ser aplicado por equipe multiprofissional, entretanto é o enfermeiro o profissional da equipe indicado para a avaliação do quadro clínico do usuário, pois apresenta habilidades de comunicação e avaliação, associadas ao conhecimento dos princípios ético-legais e técnico-científicos que regem a profissão( ${ }^{(7)}$. Nesse contexto, acolher não se restringe a uma ação atentiva e amigável do profissional. O acolhimento implica na coordenação do cuidado responsável e resolutivo, visando a romper e eliminar barreiras que dificultam ou impedem o acesso da população aos serviços. Através da classificação de risco busca-se efetivar a equidade, viabilizando o acesso aos serviços de saúde e a reorganização do processo de trabalho, possibilitando o acolhimento e a escuta, colocando a equipe na linha de frente para acolher/escutar ${ }^{(8)}$.

Evidenciou-se que a utilização desse protocolo nos serviços de assistência à saúde interferiu de forma antagônica no acesso dos usuários. Por um lado, houve aumento do tempo de espera, dificuldade do usuário em ser atendido e agendamento dos casos não agudos. Ressalta-se, no entanto, a garantia do atendimento, a porta aberta do serviço, o fim do atendimento por ordem de chegada e organização da demanda espontânea ${ }^{(9)}$.

O SUS vem, cotidianamente, buscando melhorar o acesso aos usuários e, para isso, desenvolve programas e políticas públicas, entre elas a Política Nacional de Promoção de Saúde (PNPS), criada em 2006 e redefinida em 2014, a qual propõe formar e fortalecer a transversalidade da promoção nas Redes de Atenção em Saúde (RAS), favorecendo práticas de cuidado humanizadas ancoradas na integralidade do cuidado ${ }^{(10)}$. Ademais, essa política busca avançar na articulação intersetorial e intrassetorial no tocante a: Vigilância em Saúde, Atenção Primária à Saúde (APSP) e Redes de Urgência e Emergência (RUE) do território( ${ }^{(10)}$. Assim, a RUE é entendida como uma das formas de assistência que produz saúde.

Ressalta-se a relevância deste estudo acerca do ACCR nesse serviço, porque os resultados da pesquisa poderão contribuir com o (re)planejamento da oferta do serviço de urgência /emergência, de forma a favorecer a humanização e a qualidade do cuidado.

Diante do exposto, elaborou-se a seguinte questão norteadora: qual a percepção de profissionais e usuários em relação ao acolhimento com classificação de risco (ACCR) em um serviço de urgência/emergência? Para tanto, a presente pesquisa tem como objetivo conhecer a percepção de profissionais de saúde e usuários em relação ao acolhimento com classificação de risco em um serviço de urgência/emergência.

\section{MÉTODOS}

Estudo exploratório-descritivo, com abordagem qualitativa(11), realizado na unidade de urgência/emergência de um hospital filantrópico do interior gaúcho.

Essa instituição dispõe de mais de 120 leitos, é referência em saúde na Região Noroeste do Rio Grande do Sul, Brasil, para duas coordenadorias de saúde, abrangendo aproximadamente 1.200 mil usuários. É referência regional em quatro especialidades: neurologia, traumatologia, oncologia e terapia renal, e ainda presta serviços a pacientes de diversos convênios, sendo mais de $80 \%$ provenientes do SUS ${ }^{(12)}$.

A população do estudo foi constituída por 45 profissionais de saúde que atuam na unidade de urgência/ emergência (médicos, enfermeiros e técnicos de enfermagem) e 150 usuários do serviço, que foram atendidos no período de coleta de dados (agosto e setembro de 2017).

Como critérios de inclusão, elencaram-se: ser profissional de saúde e atuar no serviço há, pelo menos, seis meses. Para os usuários, idade superior a 18 anos, possuir condição alopsíquica de responder à pesquisa e ter passado pelo ACCR. Assim, consideraram-se como critérios de exclusão: profissionais que estivessem afastados por licenças de qualquer natureza no período da coleta de dados e os usuários com limitações cognitivas ou com sequelas neurológicas que pudessem restringir a participação no momento do estudo. A amostra, então, foi composta por 15 profissionais de saúde e nove usuários.

Realizou-se o convite de forma presencial, explicando a cada um dos participantes o objetivo do estudo e, em seguida, agendou-se uma data para o início da coleta de dados, cujo instrumento foi a entrevista semiestruturada, técnica básica de coleta em estudos de natureza qualitativa ${ }^{(11)}$.

A entrevista foi composta por uma questão norteadora (como você percebe acolhimento e classificação de risco em um serviço de emergência/emergência?), tendo sido realizada de forma individual, agendada, em sala reservada no próprio serviço de saúde. Realizou-se a gravação com auxílio de um gravador digital, com duração média de 15 
minutos, assegurando-se, assim, um material fidedigno para análise. O encerramento amostral se deu quando o objetivo da pesquisa foi alcançado, levando-se em consideração o critério de saturação de dados ${ }^{(13)}$.

Para a análise dos dados, utilizou-se a técnica da análise temática ${ }^{(14)}$, que prevê três fases: pré-análise (leitura geral do material de campo, tendo em vista o pensamento crítico do pesquisador sobre o texto e o objeto em estudo), exploração do material (aproximação das unidades de significação e formar as categorias a fim de responder o objeto em estudo) e o tratamento dos resultados obtidos e interpretação (pressupõe a discussão dos dados a partir do referencial teórico, na qual foram utilizados artigos acadêmicos e legislação vigente).

Desse modo, foi possível organizar os resultados em três categorias temáticas, que tratam sobre: O cotidiano de trabalho dos profissionais em relação ao acolhimento e classificação de risco (resultado dos relatos de profissionais e usuários); Dificuldade do usuário em compreender a classificação de risco estabelecida pelos profissionais (resultado dos relatos de profissionais e usuários); Importância da capacitação para atuação em equipe no acolhimento e classificação de risco (resultado dos relatos dos profissionais).

Este estudo respeitou os aspectos éticos da pesquisa conforme ditam as diretrizes e normas regulamentadoras de pesquisa envolvendo seres humanos ${ }^{(15)}$. Os participantes assinaram o Termo de Consentimento Livre e Esclarecido e o projeto foi aprovado por Comitê de Ética em Pesquisa, com o Parecer n. ${ }^{\circ}$ 2.202.477.

Para garantia do anonimato, os participantes foram identificados por meio de um código relativo à letra inicial da categoria profissional enumerados, sequencialmente, de acordo com a ordem de realização da entrevista, conforme mostra o exemplo: médico (M1 ...), enfermeiro (E1 ...), técnico de enfermagem (TE1 ...) e usuário (U1...).

\section{RESULTADOS E DISCUSSÃO}

Neste espaço serão apresentadas e discutidas, à luz da literatura, as categorias que surgiram do estudo: 0 cotidiano de trabalho dos profissionais em relação ao acolhimento e classificação de risco, (resultado dos relatos de profissionais e usuários); Dificuldade do usuário em compreender a classificação de risco estabelecida pelos profissionais (resultado dos relatos de profissionais e usuários); Importância da capacitação para atuação em equipe no acolhimento e classificação de risco (resultado dos relatos dos profissionais).

\section{O cotidiano de trabalho dos profissionais em relação ao acolhimento e classificação de risco}

Evidenciou-se, nesta categoria, que, no processo de trabalho dos profissionais de saúde, baseado no protocolo de Manchester ${ }^{(5)}$, são considerados fatores como o estabelecimento de rotinas previamente implantadas, a disponibilidade de recursos humanos e o acesso dos usuários aos exames, o que, por vezes, acarreta sobrecarga de trabalho e demora na resolução das necessidades dos usuários, como pode ser visto nas falas a seguir:

"O fluxo depende de cada profissional. Às vezes, tem usuário que precisa de atendimento, então, classificamos, comunicamos o médico, mas ele não vem. Ou ele precisa de um parecer de outro médico, mas este demora a vir avaliar, ou depende de um exame que só é feito no final da tarde, e depois esse laudo só chega mais à noite. Então, dependendo do profissional que está fazendo o exame, do profissional que precisa vir avaliar e da disponibilidade, esse usuário vai ter um fluxo melhorou não." (E4)

Em outra fala, o médico discorreu:

"[...] tem final de semana que eu faço 36 horas de plantão, em outros faço 60 horas, atendimentos diversos. Não tenho rotina! A gente atende usuários com as mais diversas queixas, por isso é importante realizar uma classificação adequada. Não é deixar de atender, mas por um sistema de prioridades [...]." (M1)

"O acolhimento e classificação são muito importantes, tanto para a instituição quanto para o próprio usuário. Na minha opinião, ainda essa parte... está sendo mal administrada." (TE8)

A implantação do Sistema de Triagem de Manchester (STM) ocorreu há duas décadas, em vários países, e proporcionou um sistema de apoio ao enfermeiro para tomada de decisão na triagem, permitindo a realização de mudanças no cotidiano de trabalho desse profissional ${ }^{(16)}$. Esse fato reforça o impacto sobre as práticas cotidianas, uma vez que uma única categoria profissional é responsável por triar os usuários e realizar as demais atividades preconizadas para a APS ${ }^{(5)}$.

Nesse contexto, o trabalho consiste numa prática que visa a organizar o acesso dos usuários aos atendimentos, primando que sejam realizados em ordem de prioridade, pois possuem respaldo. Nessa direção, o ACCR é realizado pelo enfermeiro com auxílio do técnico de enfermagem: 
"O acolhimento e classificação de risco dá respaldo para o profissional e também para o usuário. O enfermeiro sabe que não vai fazer uma classificação aleatória do 'achar que'. Vai classificar conforme Manchester, vai dar uma cor, e essa cor, ele sabe que ela pode mudar também. Então, o usuário pode ser triado com um amarelo, mas ele pode passar para um laranja dependendo da intensidade da dor. Se houver piora acaba sendo classificado e passado antes." (E1)

"O usuário deve ser priorizado conforme a classificação. Se o usuário necessita realmente de um atendimento imediato. Deve ser avaliado o quanto aquele usuário pode aguardar. Depois que passou da porta para dentro, a gente tem que atender, não tem como mandar o usuário embora. Tem algumas coisas que conseguem ser filtradas, justamente por essa classificação." (TE8)

Identifica-se que a utilização de protocolos de classificação de risco é benéfica para usuários e equipe, pois padroniza o atendimento, diminui os riscos causados durante a espera e proporciona maior segurança para os usuários $^{(3)}$.

Outro aspecto importante, segundo a concepção dos profissionais, está relacionado ao espaço físico da instituição, que deveria oferecer melhor comodidade para profissionais e usuários, possibilitando, consequentemente, um atendimento de qualidade. Diante desses aspectos, seria importante que existisse uma sala especifica para realizar o ACCR, permitindo evitar estresse e realizar uma avaliação e classificação adequada. Sobre esse assunto, pode-se ver as falas a seguir:

"Não temos uma sala de classificação, que seria muito importante, porque ali você consegue atender melhor o usuário." (TE8)

"A parte da estrutura, o usuário pode ser classificado e ir para sala de espera. Então, seria importante ter uma porta de vidro por onde você pudesse olhar esse usuário." (E1)

Uma pesquisa avaliou a partir da perspectiva dos trabalhadores a estrutura, o processo e o resultado da implementação do instrumento Recepção com classificação de risco (RRR) (nome similar ao ACCR), e evidenciou que os principais fatores agravantes para o manejo desse instrumento são a falta de espaço físico, problemas nas relações com a equipe multidisciplinar e dificuldade em efetivar a conduta definida. Os autores concluem que se a conduta essencial proposta pelo RRR for, de fato, implementada nos serviços de emergência investigados, ainda seria necessário tornar esse sistema totalmente operacional ${ }^{(17)}$.

No estudo ora apresentado, os profissionais entrevistados apontam que a carência de equipamentos e de materiais e a estrutura física da unidade são fatores dificultadores para a realização do trabalho. Apesar disso, dedicam-se a fim de dar todo o suporte que o usuário necessita. A demanda de usuários no ACCR é grande e, em muitos momentos, a unidade mantém alguns internados em função da falta de leitos em outras unidades, o que acarreta sobrecarga nos profissionais da Enfermagem:

"Às vezes, falta material para a gente trabalhar." (TE5)

"Nós precisávamos de mais espaço para os usuários, porque aqui é um PA [pronto atendimento], às vezes tem usuário entubado, tem usuário grave. Não tem onde colocar os usuários, eles ficam nas macas superlotando a unidade. O PA não é de internação, PA é urgência e emergência. Isso atrasa, dificulta o trabalho." (TE7)

Para os enfermeiros avaliados, a prioridade é o atendimento do usuário. Tal prioridade necessita de critérios objetivos que possam nortear a dinamização do cuidado em um espaço que requer intervenções imediatas. Além disso, busca-se o vínculo com os usuários e seus familiares no acolhimento, de modo a ofertar um ambiente seguro e amenizador de angústias e sofrimentos, em que a comunicação é uma tecnologia relacional essencial:

"Eu chego, recebo o plantão, verifico os sinais dos usuários, faço evolução. Passo tudo para o arquivo deles, tiro os cartões de medicação, vou à farmácia, administro a medicação, vou atendendo as intercorrências. Vejo as consultas, as emergências que chegam e também se tem paciente com dor." (TE2)

"Eu olho tudo, faço tudo com calma, pode estar agitado, mas a prioridade são eles. Depois de tudo organizado, me detenho nos livros de registros, nos materiais para lavar, para buscar na central de materiais esterilizados." (TE7)

"Quando eu chego, vejo o que é prioridade, se tem usuário grave. Depois, eu vejo os que estão em observação. Eu sempre vou junto com os médicos, eles sempre pedem o acompanhamento da gente, e depois vou fazendo as rotinas da unidade." (E2) 
A classificação de risco nos serviços de urgência/emergência é uma atividade complexa que depende tanto das habilidades e competências dos enfermeiros quanto de fatores externos e subjetivos. Nessa direção, podem-se citar o ambiente de trabalho, o relacionamento interpessoal e a comunicação ${ }^{(16)}$ aspectos fundamentais para o adequado ACCR pelos enfermeiros.

O ACCR pode ser caracterizado como uma oportunidade de o profissional desenvolver planos de cuidado ao usuário. Para isso, precisa estar atento às possíveis alterações que possam acontecer no quadro clínico. Assim, os sinais de alerta evidentes em alguns fluxogramas e escalas validadas ajudam nessa tomada decisão(6).

Ao realizar ACCR, é fundamental que os profissionais desenvolvam uma escuta qualificada ${ }^{(18)}$, a fim de que possam emergir as reais necessidades do usuário na perspectiva do cuidado integral e resolutivo:

"O lado humano do atendimento, daquele acolhimento, de tentar se ver no usuário. Isso o que eu acho de mais positivo." (M1)

A política de humanização visa à concretização dos princípios do SUS, sendo um desafio aos profissionais da área da saúde, em particular no contexto do cuidado na urgência/ emergência. Isso se deve à alta densidade tecnológica e complexidade dessa unidade. Assim, o Ministério da Saúde elaborou o Programa Nacional de Humanização da Assistência Hospitalar (PNHAH), objetivando humanizar a assistência hospitalar oferecida aos pacientes, aproximando usuários, profissionais, hospital e comunidade, com finalidade de garantir a eficiência na qualidade e a eficácia nos serviços prestados ${ }^{(19)}$.

Nesse espaço de cuidado, é indispensável ao profissional a obtenção de habilidades e competências específicas, vinculando o saber técnicocientífico à humanização e singularidade do cuidado.

\section{Dificuldade do usuário em compreender a classificação de risco estabelecida pelos profissionais}

No entendimento dos profissionais, nesta categoria, os usuários apresentam dificuldade na compreensão do funcionamento do ACCR, visto que gostariam de ser atendidos de acordo com a ordem de chegada ao serviço. Diante disso, há a necessidade de realizar explicações periódicas a respeito dessa forma de atendimento, substituindo a 'velha máxima' de quem chega primeiro, também, é atendido primeiro:

"Hoje, uma senhora estava reclamando que chegou primeiro e foi atendida por último." (E2)

"Na maioria das vezes, não é dado ao usuário a orientação sobre a classificação, porque o fluxo é muito grande. O certo seria fazer essa classificação e orientar o usuário." (E3)

"O atendimento foi bom, só que eu gostaria que dessem mais atenção para dor. A dor que eu estou sentindo agora não tem explicação! É absurda!" (U5)

O discernimento acerca do que é, de fato, urgente entre usuários e profissionais afeta de modo bem significativo a relação entre eles. Estudo realizado em Cabo Verde revela que os usuários possuem pouco conhecimento sobre a triagem com classificação de risco, o que pode contribuir para a superlotação e prejudicar o atendimento dos casos considerados urgentes ${ }^{(20)}$. Nessa direção, estudo italiano revela que o uso de um sistema de classificação eficaz implica em correlação positiva entre a superlotação e o tempo de espera. Os autores salientam que não houve prejuízo no atendimento de usuários considerados não urgentes e também não houve influência no tempo de atendimento de casos de maior gravidade(21).

O entendimento do profissional para classificar casos urgentes e não urgentes pode ser mal interpretado pelo usuário, o qual procura a solução do problema que Ihe perturba e desconhece sua causa. Outro estudo investigou a confiabilidade do protocolo de Manchester enquanto eficiência, e demonstrou que omesmo é considerado substancial, sendo influenciada a sua resolutividade pela experiência clínica do enfermeiro. O protocolo de Manchester é um método seguro para definição das prioridades clínicas, desde que se tenha conhecimento dos diversos fluxogramas de classificação(22).

Um estudo brasileiro, entretanto, revela que a insatisfação do usuário está relacionada com a forma de organização do serviço. Entre os aspectos negativos, estão: excesso de burocracia no referenciamento para o nível terciário de atenção, demora no atendimento e falta de profissionais médicos em tempo integral ${ }^{(23)}$.

É pertinente que o usuário seja instruído quanto ao ACCR, pois, em muitas instituições, há somente um banner explicativo. Deve-se levar em consideração que alguns usuários não sabem ou não conseguem ler, ou podem apresentar dificuldades de interpretação. Assim, faz-se necessário o acolhimento e orientação sobre essa prática: 
"Eu não sei o que é isso." (U1; U7)

"Entendo que tem prioridade, mas, às vezes, o primeiro a ser atendido não é tão urgente, quanto àquele que está ali com tanta dor." (U5)

"Eu acho que é bom, mas, no meu ver, é injusto [...] Eu cheguei antes e passaram ele na frente." (U6)

"Não, não recebi, mas li o painel ali na frente." (U8)

"O acolhimento geralmente é feito pelo enfermeiro, mas, às vezes, também pelo técnico [de enfermagem]. Inicia-se o acolhimento a partir do momento que chama o paciente para verificação dos sinais vitais e anota a queixa principal." (E4)

O acolhimento pode ser efetuado por qualquer profissional de saúde, mas compete ao enfermeiro executar a classificação de risco. Destaca-se que a classificação correta do paciente, atividade de responsabilidade do enfermeiro, é o primeiro passo para garantir a segurança do paciente em serviços de urgência/emergência ${ }^{(24)}$. É considerável que essa disposição seja divulgada com clareza para os usuários ${ }^{(25)}$.

Com relação ao fluxo de usuários, alguns dos profissionais denotam que não há homogeneidade nas informações. Assim, por vezes, há uma falta de entendimento do que é o ACCR e o que ele preconiza:

"É muita demanda, e aí acaba que você não consegue ter tanto tempo. Às vezes, são quatro, cinco, dez pacientes aguardando, e aí a qualidade do acolhimento fica precarizado." (M1)

"Às vezes, a gente não consegue atender exatamente como a gente quer." (TE2)

"São usuários que se enquadram nesse conjunto, de urgência e emergência mesmo. São politraumatizados, acidente vascular cerebral..." (M3)

O ACCR, por si só, não garante a gestão adequada do tempo de espera para atendimento. Para além da classificação, é preciso que o serviço esteja organizado em fluxos assistenciais que garantam a continuidade do atendimento no serviço de urgência e em outros pontos da rede assistencial. Os fluxos de atendimento são essenciais para que o acesso ao atendimento médico e aos recursos assistenciais ocorram no tempo determinado pelo protocolo direcionador, de acordo com o nível de gravidade do usuário ${ }^{(24)}$.

O protocolo de Manchester é um preditor que orienta a necessidade de internação e risco de morte, sendo útil para gestão da assistência em saúde nos serviços de urgência/emergência, e que respalda os profissionais quanto o sequenciamento de estratégias de cuidado ${ }^{(26)}$. Aponta-se a necessidade de continuidade nas investigações cientificas para a discussão de possíveis modificações no referido protocolo para que seja utilizado com maior segurança nos serviços de urgência/emergência ${ }^{(26)}$.

A sobrecarga de trabalho e a demora do atendimento ocorrem em consequência de diversos fatores, até mesmo desde o atendimento da ESF, onde, por vezes, os usuários não são atendidos devido à ausência de médicos, sendo encaminhados para a unidade de urgência/emergência do hospital. Essa demora também tende a acarretar a insatisfação do usuário e do profissional:

"Muitas vezes, a gente chama [o médico] e ele está em outro procedimento, daí que sempre acaba sobrando para nós." (TE5)

"Logo que iniciou o acolhimento aqui na unidade, muitas pessoas se baseavam que o usuário poderia esperar duas horas, e esse é o ponto negativo." (TE6)

Observando os relatos anteriores, evidencia-se a importância do trabalho em equipe, e que o dimensionamento de pessoal possa dar conta das reais necessidades do cotidiano, como o ACCR. Além disso, vale ressaltar que, para o sucesso do atendimento, são necessários recursos humanos em quantidade e devidamente instrumentalizados para o cuidado. Essa situação pode trazer uma maior resolutividade no serviço e também a satisfação de usuários e profissionais de saúde:

"A classificação de risco é importante, porque serve para garantir que os pacientes mais graves sejam atendidos de forma mais rápida." (M2)

"Eu acho que esse acolhimento é bemfeito para poder saber o grau da gravidade do paciente." (TE5)

De acordo com os depoimentos acima, compreende-se que o tempo de atendimento aos usuários é fator decisivo para a resolução das necessidades dos usuários, para a estabilização do risco ou do quadro agudo apresentado pelo usuário. 


\section{A importância da capacitação profissional para atuação no ACCR}

Esta categoria versa sobre a importância da capacitação profissional para atuação no ACCR. É uma estratégia importante e que auxilia de maneira significativa para o desenvolvimento das ações no ACCR. É essencial que os profissionais estejam qualificados de forma a imprimir efetividade em sua atuação. Para tanto, entende-se que as instituições precisam desenvolver espaços de formação permanente, de forma a fortalecer a qualidade assistencial ofertada nos serviços de urgência/emergência:

"Às vezes, a pessoa que faz ACCR não tem uma formação adequada [e], por inexperiência ou falta de um treinamento, acaba classificando de forma errada." (M2)

"Nós deveriamos receber um treinamento mais especializado, porque nós fazemos baseado no que a gente visualiza no usuário. Quem tem essa atribuição é o enfermeiro, ele pode classificar o paciente, dizer 'o senhor tem isso e pode procurar a ESF que é a sua referência, porque, aqui, é um prontosocorro'." (TE8)

Os achados descritos anteriormente são corroborados por outro estudo em que os profissionais sentem que possuem pouco conhecimento, bem como manifestam sofrimento perante as tensões que passam ${ }^{(27)}$. Sugere-se que as instituições de saúde incorporem os conceitos da Educação Permanente em Saúde (EPS) de acordo com os princípios da problematização, a contextualização da realidade, as pedagogias inovadoras e o pensamento reflexivo.

Nessa perspectiva, a concepção problematizadora pode constituir-se num aporte para as ações educativas, indicando avanço conceitual sobre a EPS no Brasil. No entanto as ações de educação permanente, por vezes, acontecem desarticuladas do processo de trabalho. Existem dificuldades de compreensão e aplicação, com repercussões nos serviços de saúde, revelando-se como desafio o comprometimento dos profissionais e gestores ${ }^{(28)}$.

$\mathrm{Na}$ unidade avaliada para conhecimento do funcionamento do ACCR, os entrevistados mencionaram que foram realizadas palestras e, para alguns, foi dado o livro do protocolo de Manchester para que pudessem ler e tirar as dúvidas. Assim, pode-se perceber que, com o tempo, muitos profissionais ainda possuem dúvidas a respeito do acolhimento com classificação de risco e necessitam passar por um novo treinamento:

"Não recebi treinamento. A gente ia começar o Manchester, foi nos dado o livro para gente ir lendo, e foi falado algumas coisas referente a isso. Mas bem superficial, e o dia a dia, e você lendo e aprendendo e aprimorando." (E3)

"Eles explicaram como iria ser, como deveríamos agir, e tiraram algumas dúvidas. Foi bem interessante." (TE2)

A EPS é um dos instrumentos do processo de trabalho que o enfermeiro utiliza para qualificar o conhecimento técnicocientífico essencial à assistência ao usuário nas áreas de urgência/emergência. Assim, tem-se a finalidade de aprimorar as ações no que se refere à prática das habilidades na assistência de enfermagem. A equipe de enfermagem que atua em urgência/emergência sente a necessidade de EPS para ingressar nos serviços e também a percebem como uma grande aliada, facilitando o processo de trabalho. Os participantes reforçaram a ideia de que a EPS não se restringe aos conhecimentos técnicos, referindo-a como algo mais abrangente, que favorece o crescimento pessoal e organizacional(29).

A Portaria.$^{\circ}{ }^{2048^{(30)}}$ assegura que o profissional de saúde, para atuar na classificação de risco, necessita receber treinamento específico e aplicar o protocolo preestabelecido, por não exigir uma especialização específica. Acredita-se que a falta de treinamento pode ocasionar erros no processo de classificação dos usuários, o que pode gerar falsas classificações, colocando em risco a saúde da população que busca esse tipo de atendimento.

Nesse contexto, a PNPS ${ }^{(10)}$ destaca a ampliação do conceito de saúde por meio do referencial teórico da promoção da saúde. Trata-se de um conjunto de estratégias e formas de produzir saúde de forma individual e coletiva, que também se equipara aos serviços de urgência/emergência por utilizar o ACCR como uma ferramenta que norteia e organiza o fluxo e o desenvolvimento das atividades nesses serviços, impactando positivamente na saúde da coletividade.

Como implicações para a prática clínica, a investigação em tela apresenta subsídios para nortear a capacitação profissional através de programas de EPS, haja vista que apontou potencialidades e limites do ACCR na perspectiva de profissionais e de usuários. Em vista disso, recomenda-se que sejam realizados estudos de intervenção buscando potencializar as ações já desenvolvidas e superar os limites existentes para, consequentemente, promover uma melhoria na qualidade da assistência aos usuários de serviços de urgência/emergência.

Como limitação desta pesquisa tem-se que o estudo foi realizado em um único local. Contudo acredita-se que, como contribuições, este estudo possa subsidiar novos estudos e discussões que visam ao aprimoramento do dispositivo ACCR, trazendo resultados favoráveis à qualidade do atendimento em urgência/emergência. 


\section{CONSIDERAÇÕES FINAIS}

Evidenciou-se que os usuários não têm clareza a respeito dos critérios utilizados para a realização do ACCR, o que gera conflitos entre profissionais e usuários. Há necessidade de repensar as formas de esclarecer os usuários quanto à importância desse sistema.

Embora existam treinamentos, nem todos os profissionais os realizaram. Além disso, alguns, por terem sido admitidos na instituição após a implantação do ACCR, não tiveram um treinamento adequado. Assim, aponta-se a necessidade de maior investimento na capacitação e atualização desses profissionais de saúde e de readequação de recursos materiais e de infraestrutura a fim de qualificar a assistência.

\section{CONFLITOS DE INTERESSE}

Os autores declaram que não houve conflitos de interesses na realização da pesquisa.

\section{CONTRIBUIÇÕES}

Thais Santos Campos e Éder Luís Arboit contribuíram com a elaboração e delineamento do estudo; aquisição, análise e interpretação de dados; redação e/ou revisão do manuscrito. Claudelí Mistura, Cristina Thum, Jaqueline Arboit e Silviamar Camponogara contribuíram com a elaboração e delineamento do estudo e a redação e/ou revisão do manuscrito. Todos os autores aprovaram a versão final ora publicada na Revista Brasileira em Promoção da Saúde.

\section{REFERÊNCIAS}

1. Santos MN, Dantas JMM Jr. Políticas públicas e a rede de atenção às urgências. In: Santos MN, Medeiros RM, Soares OM. Emergência e Cuidados críticos para Enfermagem: conhecimentos, habilidades - atitudes. Porto Alegre: Moriá; 2018. p. 34-42.

2. Massaro IAC, Massaro A. O uso do Kan Ban na gestão do cuidado: superando limites. Rev Adm Saúde. 2017;17(66):1-8.

3. Pagliotto LF, Souza PB, Thomazini JO, Ortega ABA, Vavra SMF. Classificação de risco em uma unidade de urgência e emergência do interior paulista. Cuidarte Enferm. 2016;10(2):148-55.

4. Parenti N, Reggiani ML, lannone P, Percudani $D$, Dowding $D$. A systematic review on the validity and reliability of an emergency department triage scale, the Manchester Triage System. Int J Nurs Stud. 2014;51(7):1062-9.

5. Moreira DA, Tibães HBB, Batista RCR, Cardoso CML, Santos CS, Brito MJM. Manchester Screening System in Primary Health Care: a new hosting way? Int Arquives Med. 2015;8(261):1-9.

6. Mackway-Jones K, Marsden J, Windle J. Sistema Manchester de Classificação de Risco. $2^{\mathrm{a}}$ ed. Belo Horizonte: Grupo Brasileiro de Classificação de Risco; 2010.

7. Conselho Federal de Enfermagem (BR). Resolução Cofen $n^{\circ} 423$, de 9 de abril de 2012. Normatiza no âmbito do Sistema Cofen/Conselhos Regionais de Enfermagem, a participação do enfermeiro na atividade de classificação de riscos. Brasília: Cofen; 2012.

8. Davim RMB. Acolhimento com humanização. Rev Enferm UFPE. 2017;11(2):1-2.

9. Moreira DA, Tibães HBB, Batista RCR, Cardoso CML, Brito MJM. Manchester triage system in primary health care: ambiguities and challenges related to access. Texto \& Contexto Enferm. 2017;26(2):1-8.

10. Ministério da Saúde (BR). Portaria № 2.446/GM de 11 de Novembro de 2014. Redefine a Política Nacional de Promoção da Saúde. Brasília: Ministério da Saúde; 2014.

11. Cooper DR, Schindler PS. Métodos de pesquisa em administração. 12a ed. Porto Alegre: AMGH; 2016.

12. Hospital São Vicente de Paulo. Institucional [Internet]. 2018 [acesso em 2019 Nov 11]. Disponível em: http:// hsvpca.com.br/institucional/

13. Minayo MCS. Amostragem e saturação em pesquisa qualitativa: consensos e controvérsias. Rev Pesqui Qualitativa. 2017;5(7):1-12. 
14. Minayo MCS. O desafio do conhecimento: pesquisa qualitativa em saúde. 14ª ed. São Paulo: Hucitec; 2014.

15. Conselho Nacional de Saúde (BR). Resolução 466 de 12 de dezembro de 2012: diretrizes e normas regulamentadoras de pesquisas envolvendo seres humanos. Brasília: Ministério da Saúde; 2012.

16. Mirhaghi A, Mazlom R, Heydari A, Ebrahimi M. The Reliability of the Manchester Triage System (MTS): A Meta-analysis. J Evid Based Med. 2017;10(2):129-35.

17. Oliveira KKD, Amorim KKPS, Lima APFN, Monteiro Al. Impact of the implementation of patient engagement with risk classification for professional work of one urgent care unit. Rev Min Enferm. 2013;17(1):157-64.

18. Luiz FF, Caregnato RCA, Costa MR. Humanização na Terapia Intensiva: percepção do familiar e do profissional de saúde. Rev Bras Enferm. 2017;70(5):1095-103.

19. Ministério da Saúde (BR), Secretaria de Assistência a Saúde. Cartilha Humaniza SUS: Política Nacional de Humanização. Brasília: Ministério da Saúde; 2015.

20. Spagnuolo RS, Silva MNL, Meneguin S, Bassetto JGB, Fernandes VC. Nursing team's perceptions of humanization in intensive care. Rev Bras Promoç Saúde. 2017;30(2):249-54.

21. Cremonesi P, di Bella E, Montefiori M, Persico L. The robustness and effectiveness of the triage system at times of overcrowding and the extra costs due to inappropriate use of emergency departments. Appl Health Econ Health Policy. 2015;13(5):507-14.

22. Souza CC, Chianca TCM, Cordeiro W Jr, Rausch MCP, Nascimento GFL. Reliability analysis of the Manchester Triage System: inter-observer and intra-observer agreement. Rev Latinoam Enferm. 2018;26:e3005.

23. Machado GVC, Bento LMS, Miranda WD, Azevedo HR, Gonçalves PMS, Bonolo PF. A visão do usuário em relação à qualidade do atendimento em um serviço de urgência. Rev Med Minas Gerais. 2016;26:1-7.

24. Rodrigues APB, Ribeiro SCL, Santos AMR, Moura MEB, Mesquita GV, Brito JNPO. Concepção da equipe de enfermagem sobre acolhimento com classificação de risco. Rev Enferm UFPE. 2014;8(8):2626-32.

25. Souza CC. Atuação do enfermeiro na classificação de risco em serviços de urgência e emergência e a segurança do paciente. Rev Enferm Centro-Oeste Mineiro. 2017;7:1-2.

26. Souza CC, Araújo FA, Chianca TCM. Scientific literature on the reliability and validity of the manchester triage system (MTS) protocol: a integrative literature review. Rev Esc Enferm USP. 2015;49(1):144-51.

27. Freitas FFB, Matos GSS, Cavalcante AC, Pinheiro MBGN, Sousa MNA. O papel do enfermeiro no serviço de acolhimento e classificação de risco no setor de urgência e emergência. Rev Interdisciplin Saúde. 2015;2(3):314-33.

28. Campos KFC, Sena RR, Silva KL. Permanent professional education in healthcare services. Esc Anna Nery. 2017;21(4):1-10.

29. Piazza M, Ramos BJ, Oliveira SN, Prado ML, Massaroli A, Zamprogna KM. Educação permanente em unidades de pronto atendimento 24 horas: necessidade e contribuição à enfermagem. $J$ Nurs Health. 2015;5(1):47-54.

30. Ministério da Saúde (BR). Portaria No 2048/GM de 05 de novembro de 2002. Aprova, na forma do Anexo desta Portaria, o Regulamento Técnico dos Sistemas Estaduais de Urgência e Emergência. Brasília: Ministério da Saúde; 2002. 
Endereço do primeiro autor:

Thais Santos Campos

Hospital São Vicente de Paulo - Cruz Alta

Av. Venâncio Aires, 163

Bairro: Centro

CEP: 98025-790 - Cruz Alta - RS - Brasil

E-mail: taia.553@gmail.com

Endereço para correspondência:

Éder Luiz Arboit

Universidade de Cruz Alta - UNICRUZ

Rodovia Municipal Jacob Della Mea, s/n km 5,6 - Parada Benito

CEP: 98020-290 - Cruz Alta - RS - Brasil

E-mail: earboit@unicruz.edu.br

Como citar: Campos TS, Arboit EL, Mistura C, Thum C, Arboit J, Camponogara S. Acolhimento e classificação de risco: percepção de profissionais de saúde e usuários. Rev Bras Promoç Saúde. 2020;33:9786. 